PAPER

\title{
Does the risk of developing dementia increase with the number of episodes in patients with depressive disorder and in patients with bipolar disorder?
}

\author{
L V Kessing, P K Andersen
}

J Neurol Neurosurg Psychiatry 2004;75:1662-1666. doi: 10.1136/jnnp.2003.031773

See end of article for authors' affiliations

....................

Correspondence to:

L V Kessing, Department of

Psychiatry, University

Hospital of Copenhagen,

Rigshospitalet,

Blegdamsvej 9, DK 2100

Copenhagen $\varnothing$, Denmark;

lars.kessing@rh.dk

Received 4November 2003

In revised form

23 February 2004

Accepted 6 March 2004
Objective: Several findings suggest that some patients with depressive or bipolar disorder may be at increased risk of developing dementia. The present study aimed to investigate whether the risk of developing dementia increases with the number of affective episodes in patients with depressive disorder and in patients with bipolar disorder.

Methods: This was a case register study including all hospital admissions with primary affective disorder in Denmark during 1970-99. The effect of the number of prior episodes leading to admission on the rate of readmission with a diagnosis of dementia following the first discharge after 1985 was estimated. A total of 18726 patients with depressive disorder and 4248 patients with bipolar disorder were included in the study.

Results: The rate of a diagnosis of dementia on readmission was significantly related to the number of prior affective episodes leading to admission. On average, the rate of dementia tended to increase 13\% with every episode leading to admission for patients with depressive disorder and $6 \%$ with every episode leading to admission for patients with bipolar disorder, when adjusted for differences in age and sex. Conclusion: On average, the risk of dementia seems to increase with the number of episodes in depressive and bipolar affective disorders.
$\mathrm{F}$ indings from epidemiological studies suggest that depression or depressive symptoms may be a risk factor for development of cognitive decline or dementia (for reviews see references 1,2 ). Thus, several prospective community studies suggest that depressive symptoms or depression at baseline are associated with increased risk of developing cognitive decline at follow up ${ }^{3-10}$ although other studies have failed to confirm these findings. ${ }^{11-13}$ Additionally, some retrospective studies of patients with Alzheimer's disease suggest that a history of depression may be associated with increased risk of developing late onset Alzheimer's disease ${ }^{314-16}$ and some uncontrolled follow up studies of patients with depression suggest that subgroups of patients with depression are at increased risk of developing dementia. ${ }^{17-21}$ Finally, in two controlled (historical), prospective long term studies of patients with affective disorder we found that patients previously discharged from psychiatric hospitals with a diagnosis of single or recurrent depression or with bipolar disorder had increased risk of getting a diagnosis of dementia on readmission compared with patients with a diagnosis of neurosis and compared with the general population $^{22}$ or patients with a diagnosis of osteoarthritis or diabetes. ${ }^{23}$

The more precise nature of such a putative association between depression and dementia, however, has seldom been studied. Very few studies have investigated how the course of illness of affective disorder may affect the risk of developing cognitive decline or dementia. In one study, the number of depressive episodes was found to be associated with the severity of cognitive impairment, ${ }^{5}$ and conversely in another study, persistent but not episodic depressive episodes were found to be associated with cognitive decline..$^{10}$ Additionally, a few cross-sectional studies of patients who were investigated in the euthymic phase of affective disorder found that the number of prior affective episodes was associated with the severity of cognitive dysfunction ${ }^{24-27}$ but other studies have not confirmed this finding. ${ }^{28}$
Although the literature is sparse, we hypothesised that the number of prior affective episodes (depressive or manic) is associated with increased risk of developing dementia. The aim of the present study was to investigate whether the number of prior admissions for patients hospitalised with depressive or bipolar disorder predicted increased risk of subsequently getting readmitted with a diagnosis of dementia using a national case register of all admissions to psychiatric wards.

Only patients who had been hospitalised at least once on a psychiatric ward and only affective episodes leading to admission were included in the study; these patients thus represent the more severely influenced proportion of patients. Data on treatment or outpatient status were not available.

\section{METHODS}

\section{The register}

In Denmark, all psychiatric admissions have been registered in a nationwide register for the 5.1 million inhabitants. ${ }^{29}$ All inhabitants of Denmark have a unique person identification number that can be logically checked for errors, so it can be established with great certainty if a patient has been admitted previously, irrespective of changes in name etc. Censoring due to death and causes of death can also be established with equal certainty as the same identification number is used across all public registration systems.

Between 1 April 1970 and 31 December 1993 the International Classification of Diseases (ICD), 8th revision ${ }^{30}$ was used in Denmark. For various reasons, and to achieve better diagnostic reliability over time, it was decided not to change to the ICD, 9th revision in 1978. Since 1 January 1994 the ICD- $10^{31}$ has been used.

\section{The sample}

Patients who had had their first ever discharge in the period between 1 April 1970 and 31 December 1999 were identified

Abbreviation: ICD, International Classification of Diseases 
from the register. Patients were included in the study at the time of first discharge from a psychiatric hospital after 1 January 1986 provided that they, at their first ever discharge after 1 April 1970, had got a diagnosis of affective disorder and provided that their main diagnosis was not of organic psychosis (ICD-8 codes, 290-294 and ICD-10, code DF00-09) or schizophrenia (ICD-8, code 295 and ICD-10, code DF20-29) at other discharges before 1 January 1986. A diagnosis of affective disorder included depressive episode/ recurrent depression (ICD-8, codes 296.09 and 296.29, and ICD-10, code DF32-33.9) and manic, mixed episode/bipolar disorder (ICD-8, codes 296.19 and 296.39, and ICD-10, codes DF30-31.9 and DF38.00). Patients were classified as having bipolar disorder if they had had a diagnosis of manic, mixed episode/bipolar disorder before their date of entry into the study and as having depressive disorder if they had not had a diagnosis of manic, mixed episode/bipolar disorder. Patients were also classified according to their number of affective episodes in the period between 1 April 1970 and their entry into the study.

\section{Statistical analysis}

The hazard ratio (HR) of the number of episodes leading to admission on the rate of readmission with a discharge diagnosis of dementia was estimated using a Cox proportional hazards regression model. Time to dementia was censored if patients died, emigrated, or were not diagnosed as having dementia on readmission before 31 December 1999. The hazard ratio was adjusted for differences in sex, age, and calendar time at the first discharge date following 1985. The likelihood ratio test was used to assess whether the number of episodes leading to admission could be included as an ordinal variable and to compare the effect of episodes leading to admission for patients with depressive disorder and patients with bipolar disorder.

The model assumptions of proportional hazards between depressive and bipolar patients with different number of episodes leading to admission were checked graphically using log-log survival plots, and numerically by entering appropriate time dependent covariates.

\section{RESULTS}

During the study period from 1970 to 1999,40106 patients were diagnosed with a manic-depressive psychosis (ICD-8) or affective disorder (ICD-10) at first discharge. Among these patients, the study population was defined as the 18726 patients with depressive disorder and 4248 patients with bipolar disorder who were admitted at least once between 1986 and 1999. Following entry into the study, 337 (1.8\%) of the patients with depressive disorder and $97(2.3 \%)$ of the patients with bipolar disorder got a diagnosis of dementia at discharge from a subsequent readmission. Further characteristics of the patients included are presented in table 1 .

The results of the Cox regression analysis of the effect of the number of prior affective episodes leading to admission, adjusted for the effect of age, sex, and calendar time, are presented in table 2. Data were missing for 186 patients $(0.8 \%)$ of the total sample of 22974 patients leaving 22788 patients for the analyses. In the model, no significant effect of sex was found (HR 1.09, 95\% CI 0.89 to 1.35) but the rate of dementia increased with age (HR 1.06, 95\% CI 1.048 to $1.064)$ - that is, a $6 \%$ increase with every year. Patients with one manic episode $(n=2187)$ had a $1.46(95 \%$ CI 1.01 to 2.13) times increased rate of dementia compared with patients with one depressive episode $(n=15085)$.

First, the number of episodes was included as a categorical variable separately for patients with depressive disorder and patients with bipolar disorder. Patients with one episode were chosen as the reference group. The hazard ratios
Table 1 Characteristics of the patients at the index episode

\begin{tabular}{lll}
\hline & $\begin{array}{l}\text { Depressive } \\
\text { disorder }\end{array}$ & $\begin{array}{l}\text { Bipolar } \\
\text { disorder }\end{array}$ \\
\hline $\begin{array}{l}\text { No. of patients } \\
\text { Female sex (\%) }\end{array}$ & 18726 & 4248 \\
$\begin{array}{l}\text { Age in years, (mean (SD)) } \\
\text { Proportion with dementia } \\
\text { during follow up (\%)* }\end{array}$ & 67.4 & 59.4 \\
$\begin{array}{l}\text { Proportion dead during } \\
\text { follow up (\%)† }\end{array}$ & 1.8 & $52.0(17.3)$ \\
\hline
\end{tabular}

*Proportion of patients who had a diagnosis of dementia at readmission following the index episode.

†Proportion of patients censored because of death after the index episode.

presented thus indicate the rate of dementia for patients with a given number of prior episodes leading to admission compared with the rate for patients with one episode. Patients with two prior depressive episodes leading to admission had the same rate of dementia (HR 1.00, 95\% CI 0.25 to 4.06 ) as patients with one prior episode. Patients with three prior depressive episodes had a 2.89 times increased rate of dementia (95\% CI 0.64 to 13.02 ) compared with patients with one prior depressive episode, etc. For bipolar patients, patients with two manic episodes and patients with three manic episodes did not have increased rate of dementia compared with patients with one depressive episode, whereas patients with four or more episodes had increased rate of dementia. The rate of dementia varied significantly with the number of episodes for patients with depressive disorder $\left(\chi^{2}=27.0, \mathrm{df}=4 ; \mathrm{p}<0.001\right)$ and for patients with bipolar disorder $\left(\chi^{2}=31.9, \mathrm{df}=4 ; \mathrm{p}<0.001\right)$. The difference between the effects of episodes in depressive disorder and bipolar disorder was significant according to a likelihood ratio test $\left(\chi^{2}=11.4, \quad \mathrm{df}=4 ; \mathrm{p}<0.05\right)$. The proportional hazards assumption was found to be reasonably fulfilled.

Secondly, the number of episodes leading to admission was included as an ordinal variable. In this model, the hazard ratios indicate the factor change in the rate of dementia for each affective episode. Patients with depressive disorder and patients with bipolar disorder were considered separately. The episode number significantly predicted the rate of subsequent dementia: for every new episode leading to admission the rate increased on average 13\% (95\% CI 9\% to $16 \%$ ) for patients with depressive disorder and 6\% (95\% CI

\begin{tabular}{|c|c|c|c|c|}
\hline & \multicolumn{2}{|c|}{ Depressive disorder } & \multicolumn{2}{|c|}{ Bipolar disorder } \\
\hline & No. & $\begin{array}{l}\text { Hazard ratio } \\
(95 \% \mathrm{Cl})\end{array}$ & No. & $\begin{array}{l}\text { Hazard ratio } \\
(95 \% \mathrm{Cl})\end{array}$ \\
\hline 1 episode* & 15085 & 1 (reference) & 2187 & 1 (reference) \\
\hline 2 episodes & 1324 & $\begin{array}{l}1.00 \\
(0.25 \text { to } 4.06)\end{array}$ & 366 & $\begin{array}{l}0.34 \\
(0.05 \text { to } 2.56)\end{array}$ \\
\hline 3 episodes & 735 & $\begin{array}{l}2.89 \\
(0.64 \text { to } 13.02)\end{array}$ & 323 & $\begin{array}{l}0.80 \\
(0.15 \text { to } 4.43)\end{array}$ \\
\hline 4 episodes & 459 & $\begin{array}{l}2.70 \\
(0.58 \text { to } 12.58)\end{array}$ & 243 & $\begin{array}{l}2.05 \\
(0.39 \text { to } 10.68)\end{array}$ \\
\hline$\geqslant 5$ episodes & 983 & $\begin{array}{l}6.16 \\
(1.39 \text { to } 27.22)\end{array}$ & 1083 & $\begin{array}{l}3.19 \\
(0.69 \text { to } 14.75)\end{array}$ \\
\hline $\begin{array}{l}\text { Type of } \\
\text { disordert }\end{array}$ & & 1 (reference) & & $\begin{array}{l}1.46 \\
(1.01 \text { to } 2.13)\end{array}$ \\
\hline
\end{tabular}

*Hazard ratio: the rate of a diagnosis of dementia for patients with a given number of prior episodes compared with the rate for patients with one prior episode.

tThe effect of the type of disorder for patients with one episode. All hazard ratios are adjusted for the effects of sex, age, and calendar time. 
$3 \%$ to $10 \%$ ) for patients with bipolar disorder. To explore whether these associations also apply to patients with an interval of one year or more between the index episode and dementia, the analysis was repeated including only those cases in which time under risk was greater than one year. Similar significant effects were found (depressive disorder: $11 \%$ (95\% CI: $6 \%$ to $17 \%$ ); bipolar disorder: $8 \%$ (95\% CI 3\% to $13 \%)$ ). However, a likelihood ratio test suggested that the number of episodes expressed as an ordinal variable gave an inferior fit to the data than a categorical variable $\left(\chi^{2}=17.0\right.$, $\mathrm{df}=6 ; \mathrm{p}<0.01)$ and therefore these should be taken as a rough indication of a general increasing trend.

To explore whether the probability of getting a diagnosis of dementia simply increased with the number of admissions, the proportion of patients with a diagnosis of dementia at each admission was calculated. Among all patients admitted twice, $10.1 \%$ had a diagnosis of dementia at the second admission. The corresponding proportions were $16.1 \%$ for patients admitted three times and $15.8 \%$ for patients admitted four times. Among patients admitted five times or more, $9.3 \%$ got a diagnosis of dementia at the fifth or at a later admission. Thus, no systematic association was found between the number of admissions and the proportion of patients who were diagnosed as having dementia.

\section{DISCUSSION}

The present study showed that the risk of getting a diagnosis of dementia at readmission was affected significantly by the number of prior episodes leading to admission in depressive disorder and bipolar disorder. The effect of episodes leading to admission was significantly greater for patients with depressive disorder than for patients with bipolar disorder. The rate of dementia increased on average 13\% (95 CI 9\% to $16 \%)$ with every episode leading to admission for patients with depressive disorder and 6\% (95 CI 3\% to 10\%) with every episode leading to admission for bipolar patients.

\section{Methodological considerations}

It should be acknowledged that the study only included patients who had been hospitalised at least once and that affective episodes were included only if they resulted in hospitalisation and that the outcome (a diagnosis of dementia) only related to patients who were readmitted. The latter is illustrated by the relatively low incidence of dementia found in the study. Thus, patients may have been diagnosed as having dementia in places outside psychiatric and somatic wards following first admission without this being recorded in the registers used as the source for data for the present study.

\section{Validity of the diagnoses of affective disorders}

The diagnoses in the register are made by different clinicians throughout Denmark and are not standardised for research purposes. Diagnostic shifts due to transfer to ICD-9 are avoided, since ICD-8 was used in Denmark until ICD-10.

The validity of the affective diagnoses has been found to be good in a validity study of the register. ${ }^{32}$ In a random subsample of 100 patients from the register with clinical ICD-8 diagnoses of manic-depressive psychosis at first admission, 95 patients received a lifetime ICD-10 diagnosis of affective disorder according to research diagnostic criteria.

Part of the problem with the diagnostic validity is the diagnostic concordance between ICD-8 and ICD-10. From the present register, the concordance between the two diagnostic systems has been found to be as high as $84 \%$ for patients with affective disorder and $87 \%$ for patients with dementia. ${ }^{33}$ Thus, $13 \%$ of patients who received an ICD-8 diagnosis of dementia received an ICD-10 diagnosis of another organic mental disorder or alcohol induced mental disorder (F06-F10).
Misclassification of the diagnosis of dementia

It is well known that depression may be misclassified as dementia when symptoms of cognitive dysfunction are prominent-that is, some of the diagnoses of dementia in the present study in reality represent a pseudodemented state. However, pseudodementia is in general milder and more fluctuating in nature, while diagnoses of dementia that are given on discharge from psychiatric departments following admission for days or weeks, presumably describe a moderate to severe, progressive, demented condition. One may thus presume that the risk of misclassification is low. To investigate this further, 30 patients with primary affective disorder and a subsequent main or auxiliary diagnosis of dementia were randomly selected from the register (Kessing, unpublished data). The majority of these patients scored within the demented range on two different scales of cognitive assessment (70\% of patients scored below 100 on the CAMCOG (Cambridge Cognitive Examination) rating scale (range 0-120) ${ }^{34}$ with an average of 89.5 (SD 19.7) and $96.2 \%$ of patients scored below 1 on the Global Deterioration Scale $^{35}$ (range 1-6) with an average of 3.9 (1.0)). One may argue that patients who eventually got a diagnosis of dementia might have had several admissions previously at which dementia might have been misdiagnosed as depression. This possibility did, however, not explain our results as additional analyses revealed that the association between the number of episodes leading to admission and the risk of dementia was also found among patients with more than one year between last admission for depression and admission with a diagnosis of dementia. Anyway, it should be acknowledged that there is a need for validation of the diagnosis of dementia through criterion based diagnoses made at repeated assessments over time.

\section{Detection bias}

We find no reason to believe that doctors in psychiatric wards may be more observant of symptoms of dementia in patients who have been admitted with depression or mania many times than in patients who have seldom been admitted.

On the other hand, it has previously been found that the risk of getting readmitted increases with the number of prior episodes leading to admission for patients with affective disorders in Denmark. ${ }^{36}{ }^{37}$ This may by itself increase the probability for patients with many prior affective episodes of getting a diagnosis of dementia at admission as these patients have a greater chance of seeing a doctor in a psychiatric ward than patients with fewer admissions. However, arguing against this possibility, it was not found that the probability of getting a diagnosis of dementia simply increased with the number of admissions (the proportion of patients who got a diagnosis of dementia at the fifth or at a later admission was less than the proportion who got a diagnosis of dementia at the second, third, or fourth admission, respectively). Thus, we do not believe that such putative detection bias explain our finding of an association between the number of affective episodes and the risk of dementia.

\section{Possible confounders}

It is unlikely that the demonstrated association between affective disorder and dementia was a result of intracranial infection, cerebral arteriosclerosis, cerebrovascular accidents as stroke, haemorrhage, epilepsy, intracranial neoplasm, degenerative diseases of the central nervous system, brain trauma, endocrine disorders, metabolic and nutritional disorders, systemic infections, drug or poison intoxication, or other physical conditions, since dementia related to these disorders was coded elsewhere in ICD-8 (code 292.0 - 294.9) and ICD-10 (code DF00-09). Additionally, patients who, 
following the first discharge, at a subsequent admission received a discharge diagnosis of brain disorders other than dementia were censored.

Also, in our previous studies we found that the association between affective disorder and dementia could not be explained by alcoholism or other substance abuse..$^{22}$

\section{Conclusion}

Although we cannot exclude methodological considerations, we do not believe that the finding of an increasing risk of getting a diagnosis of dementia with the number of affective episodes leading to hospitalisation is simply a result of methodological pitfalls.

With regard to longitudinal studies of depressive disorder, our findings are in accordance with the finding of an association between the number of depressive episodes and the risk of developing Alzheimer's disease in the follow-up study by Andersen ${ }^{5}$ but contrast with the finding in the four year study by Paterniti et al. ${ }^{10}$ It is possible that a follow up time of four years is too short, as suggested by the studies of Speck et $a l^{3}$ and Palsson et $a l^{8}$ depression may act as a predictor of dementia particularly when there is a large interval between onset of depression and onset of dementia ( 10 years or more between the onset of depression and the onset of dementia). It is further possible that the association is more pronounced or is found only in patients with severe affective disorder for example as reflected by the need of hospitalisation as in the present study.

No prior longitudinal study of bipolar disorder has investigated the association between the number of episodes and the risk of developing dementia.

\section{Implications}

Data on treatment were not available in the present study. If treatment should explain the finding of an effect of the number of affective episodes on the risk of developing dementia, then this treatment should be given continuously for long periods of time for both patients with depressive disorder and patients with bipolar disorder. Antidepressants are usually only given for shorter periods in patients with bipolar disorder, ${ }^{38}$ however anxiolytics may often be given to both patient groups for a longer time. As indicated by Jorm, the literature is inconsistent as benzodiazepine use has been associated with cognitive decline ${ }^{39}$ as well as a lower incidence of Alzheimer's disease. ${ }^{40}$

The study supports the possibility of a direct association between affective disorder and dementia without any intermittent cerebral disorder ${ }^{12}$ and further supports the hypothesis that affective episodes may cause permanent affection of the brain (the scar hypothesis as suggested by Post and others ${ }^{41}$ ). If our results can be confirmed in future studies they underscore the importance of early and sustained prophylaxis of the evolving process of the illness in depressive and bipolar disorders. ${ }^{41}$ The pathophysiology behind such a direct association is unknown, although the hypothesis that affective disorder may cause dementia through a glucocorticoid cascade is gaining interest. ${ }^{42}$ Subdiagnoses of dementia were not investigated in the present study, since it is difficult clinically to distinguish betweenfor example, Alzheimer's disease (code 290.10) and dementia senilis (code 290.09) —and since, especially early in the observation period, there was seldom access to single photon emission computed tomography and magnetic resonance scanning.

Our findings need to be replicated in prospective studies involving large groups of patients followed over many years with regular assessments of the cognitive function. Further research, including brain imaging studies, within this field of affective disorder and dementia may provide further understanding of the pathophysiology underlying these disorders.

\section{Authors' affiliations}

L V Kessing, Department of Psychiatry, University of Copenhagen, Rigshospitalet, Copenhagen, Denmark

P K Andersen, Department of Biostatistics, University of Copenhagen and Department of Psychiatric Demography, University of Aarhus, Psychiatric Hospital, Risskov, Denmark

This study was supported by the Stanley Medical Research Institute and Lundbeck Foundation.

Competing interests: none declared

\section{REFERENCES}

1 Jorm AF. Is depression a risk factor for dementia or cognitive decline? A review. Gerontology 2000;46:219-27.

2 Jorm AF. History of depression as a risk factor for dementia: an updated review. Aust N Z J Psychiatry 2001;35:776-81.

3 Speck CE, Kukull WA, Brenner DE, et al. History of depression as a risk factor for Alzheimer's disease. Epidemiology 1995;6:366-9.

4 Devanand DP, Sano M, Tang MX, et al. Depressed mood and the incidence of Alzheimer's disease in the elderly living in the community. Arch Gen Psychiatry 1996:53:175-82.

5 Andersen K. Risk factors for dementia. Faculty of Health Sciences, Odense University, Denmark, 1997.

6 Yaffe K, Blackwell T, Gore R, et al. Depressive symptoms and cognitive decline in nondemented elderly women: a prospective study. Arch Gen Psychiatry 1999;56:425-30

7 Chen P, Ganguli M, Mulsant BH, et al. The temporal relationship between depressive symptoms and dementia: a community-based prospective study. Arch Gen Psychiatry 1999;56:261-6.

8 Palsson S, Aevarsson O, Skoog I. Depression, cerebral atrophy, cognitive performance and incidence of dementia. Population study of 85 -year-olds. Br J Psychiatry 1999; 174:249-53.

9 Geerlings MI, Schoevers RA, Beekman AT, et al. Depression and risk of cognitive decline and Alzheimer's disease. Results of two prospective community-based studies in The Netherlands. Br J Psychiatry 2000;176:568-75.

10 Paterniti S, Verdier-Taillefer MH, Dufouil C, et al. Depressive symptoms and cognitive decline in elderly people. Longitudinal study. Br J Psychiatry 2002;181:406-10.

11 Bickel H, Cooper B. Incidence and relative risk of dementia in an urban elderly population: findings of a prospective field study. Psychol Med 1994;24:179-92.

12 Dufouil C, Fuhrer R, Dartigues JF, et al. Longitudinal analysis of the association between depressive symptomatology and cognitive deterioration. Am J Epidemiol 1996;144:634-41.

13 Henderson AS, Korten AE, Jacomb PA, et al. The course of depression in the elderly: a longitudinal community-based study in Australia. Psychol Med 1997;27:119-29.

14 Jorm AF, van Duiin $C M$, Chandra $V$, et al. Psychiatric history and related exposures as risk factors for Alzheimer's disease: a collaborative re-analysis of case-control studies. EURODEM Risk Factors Research Group. Int J Epidemiol 1991;20(suppl 2):S43-S47.

15 Steffens DC, Plassman BL, Helms MJ, et al. A twin study of late-onset depression and apolipoprotein E epsilon 4 as risk factors for Alzheimer's disease. Biol Psychiatry 1997;41:851-6.

16 Green RC, Cupples LA, Kurz A, et al. Depression as a risk factor for Alzheimer disease-The MIRAGE study. Arch Neurol 2003;60:753-9.

17 Rabins PV, Merchant A, Nestadt G. Criteria for diagnosing reversible dementia caused by depression: validation by 2-year follow-up. Br J Psychiatry 1984; 144:488-92.

18 Pearlson GD, Rabins PV, Kim WS, et al. Structural brain CT changes and cognitive deficits in elderly depressives with and without reversible dementia ("pseudodementia"). Psychol Med 1989;19:573-84.

19 Alexopoulos GS, Meyers BS, Young RC, et al. The course of geriatric depression with "reversible dementia": a controlled study. Am J Psychiatry 1993;150:1693-9

20 Stoudemire A, Hill CD, Morris R, et al. Long-term affective and cognitive outcome in depressed older adults. Am J Psychiatry 1993;150:896-900.

21 Stoudemire A, Hill CD, Morris R, et al. Improvement in depression-related cognitive dysfunction following ECT. J Neuropsychiatry Clin Neurosci 1995;7:31-4.

22 Kessing LV, Olsen EW, Mortensen PB, et al. Dementia in affective disorder: a case-register study. Acta Psychiatr Scand 1999;100:176-85.

23 Kessing LV, Nilsson FM. Increased risk of developing dementia in patients with major affective disorders compared to patients with other medical illnesses. J Affect Disord 2003;73:261-9.

24 Kessing LV, Dam H, Joergensen OS, et al. Cognitive impairment in affective disorders. Relation to illness characteristics. Nord J Psychiatry 1996;50:305-16.

25 Kessing LV. Cognitive impairment in the euthymic phase of affective disorder. Psychol Med 1998;28:1027-38. 
26 Tham A, Engelbrektson K, Mathe AA, et al. Impaired neuropsychological performance in euthymic patients with recurring mood disorders. J Clin Psychiatry 1997; 58:26-9.

27 Van Gorp WG, Altshuler L, Theberge DC, et al. Cognitive impairment in euthymic bipolar patients with and without prior alcohol dependence. A preliminary study. Arch Gen Psychiatry 1998;55:41-6

28 Ferrier IN, Stanton BR, Kelly TP, et al. Neuropsychological function in euthymic patients with bipolar disorder. Br J Psychiatry 1999:175:246-51

29 Munk-Jorgensen P, Mortensen PB. The Danish Psychiatric Central Register. Dan Med Bull 1997;44:82-4.

30 Danish National Board of Health (Sundhedsstyrelsen). Classification of diseases. The Danish edition of ICD-8. Copenhagen: Danish National Board of Health, 1971.

31 World Health Organization. The ICD-10 classification of mental and behavioural disorders: clinical descriptions and diagnostic guidelines. Geneva: World Health Organization, 1992.

32 Kessing LV. Validity of diagnoses and other register data in patients with affective disorder. Eur Psychiatry 1998;13:392-8.

33 Kessing LV. A comparison of ICD-8 and ICD-10 diagnoses of affective disorder-a case register study. Eur Psychiatry 1998;13:342-5.
34 Roth M, Thym E, Mountioy CQ, et al. CAMDEX. A standardised instrument for diagnosis of mental disorder in the elderly with special reference to the early detection of dementia. Br J Psychiatry 1986;149:698-709.

35 Reisberg B, Ferris SH, Deleon MJ, et al. Global Deterioration Scale (GDS). Psychopharmacol Bull 1988;24:661-3.

36 Kessing LV, Andersen PK, Mortensen PB, et al. Recurrence in affective disorder. I. Case register study. Br J Psychiatry 1998;172:23-8.

37 Kessing LV, Olsen EW, Andersen PK. Recurrence in affective disorder: analyses with frailty models. Am J Epidemiol 1999;149:404-11.

38 Frances AJ, Kahn DA, Carpenter D, et al. The Expert Consensus Guidelines for treating depression in bipolar disorder. J Clin Psychiatry 1998;59(suppl 4): 73-9.

39 Prince $M$, Rabe-Hesketh S, Brennan P. Do antiarthritic drugs decrease the risk for cognitive decline? An analysis based on data from the MRC treatment trial of hypertension in older adults. Neurology 1998;50:374-9.

40 Fastbom J, Forsell Y, Winblad B. Benzodiazepines may have protective effects against Alzheimer disease. Alzheimer Dis Assoc Disord 1998;12:14-17.

41 Post RM. Transduction of psychosocial stress into the neurobiology of recurrent affective disorder. Am J Psychiatry 1992;149:999-1010.

42 O'Brien JT. The "glucocorticoid cascade" hypothesis in man. Br J Psychiatry 1997;170:199-201

\section{HISTORICAL NOTE}

doi: 10.1136/jnnp.2003.029009

\section{From orthostatic hypotension to Shy-Drager syndrome}

S yncope derives from the Greek synkoptein, meaning to strike, cut off, or weary. Hippocrates and biblical texts describe victims of fainting. In the USA syncope accounts for 3\% of emergency room visits and $1-6 \%$ of all hospital admissions, ${ }^{1}$ Pierre Adolph Piorry ${ }^{2}$ (1794-1879) in 1826 reported,

"When a patient faints, symptoms improve when he is laid flat."

Piorry was a pioneer of percussion and pleximetry, best remembered for his work in chest diseases and for coining the term "uraemia".

Thomas Addison, when describing postural syncope in adrenal failure, also noted:

"Attacks of giddiness and dimness of sight... would occur always when in the standing posture and were instantly relieved by sitting or lying down."

Bradbury and Eggleston ${ }^{4}$ first recorded a recurrent fainting of unknown cause in 1925 in three patients subject to attacks of idiopathic orthostatic hypotension. Many lone and familial cases have subsequently appeared in the literature. ${ }^{5}$

Orthostatic hypotension in association with other neurological disease occurs in tabes, syringomyelia, spinal cord injury, and in polyneuropathies, Parkinsonism, multiple system atrophy (MSA), and a variety of cerebrovascular and tumourous lesions. In 1960 Milton Shy at the NIH and Glen Albert Drager of Houston described comprehensively the clinicopathology of MSA:

"A neurological syndrome associated with orthostatic hypotension... which is of adult onset and consists of orthostatic hypotension, bladder and bowel incontinence, anhidrosis, iris atrophy, amyotrophy, ataxia, rigidity and tremor; intellect is unaffected" ${ }^{\prime \prime 6}$

Schatz later revised ${ }^{7}$ the nosology for autonomic disorders: (1) primary, autonomic failure (idiopathic orthostatic hypotension or Bradbury-Eggleston syndrome) with no neurologic defects other than autonomic dysfunction; (2) multiple system atrophy, equivalent to Shy-Drager syndrome (a sporadic, progressive, adult onset disorder characterised by autonomic dysfunction, parkinsonism, and ataxia in any combination); and (3) secondary autonomic failure delineates diseases in which the cause is known (for example diabetes, amyloidosis, dopamine beta-hydroxylase deficiency, and drug side effects).

The American Autonomic Society has defined multiple system atrophy as:

"A sporadic, progressive, adult onset disorder characterized by autonomic dysfunction, Parkinsonism, and ataxia (a failure of muscular coordination) in any combination....The main features are: Parkinsonism, Cerebellar signs, Autonomic Failure, Striatonigral Degeneration, Sporadic Olivo-ponto-cerebellar Atrophy, Shy-Drager Syndrome."

George Milton Shy (1919-1967) qualified in Oregon in 1943 and after brief war service, in which he was wounded, studied neurology at the Montreal Neurological Institute, and at the National Hospital, Queen Square. Unusually for an American, he became MRCP London by examination, in 1947. He carried out highly regarded research on myopathies and published extensively. His work on the Kearns-Sayre syndrome, oculopharyngeal myopathy (Shy-Gonatas syndrome), and a myopathy of infants (Shy-Magee syndrome) are lasting monuments to his protean interests. In 1953 he became clinical director of the National Institute of Neurological Diseases and Blindness, Bethesda, and in 1962 chairman of neurology in Pennsylvania, before taking the chair and directorship of the New York Neurological Institute, Columbia in 1967. Aged only 47 he died suddenly a few weeks later.

Little is recorded about Drager (1917-1967), a talented physician at Baylor College of Medicine, Houston, who also died young, in the same year as Shy.

J M S Pearce 304 Beverley Road, Anlaby, East Yorkshire HU10 7BG, UK jmspearce@freenet.co.uk

\section{References}

1 Maisel WH, Stevenson WG. Syncope-getting to the heart of the matter. New Engl J Med 2002;347:931-3.

2 Sakula A. Pierre Adolph Piorry (1794-1879): pioneer of percussion and pleximetry. Thorax 1979;34:575-581

3 Piorry PA. Traité de diagnostic et de séméiologie. Paris: Pur chef, 1840.

4 Bradbury S, Eggleston C. Postural hypotension: a report of three cases. J Am Heart J 1925; 1:73-86.

5 Lewis P. Familial orthostatic hypotension. Brain 1964;87:719-27.

6 Shy GM, Drager GA. A neurological syndrome associated with orthostatic hypotension: a clinical-pathologic study. Arch Neurol 1960;2:511-527.

7 Schatz IJ. Farewell to the 'Shy-Drager syndrome'. Ann Int Med 1996; 125:74-75 